\title{
SATISFAÇÃO DOS USUÁRIOS COM SERVIÇOS DA FARMÁCIA COMUNITÁRIA: UMA ABORDAGEM ESPACIAL
}

\section{USERS OF SATISFACTION WITH COMMUNITY PHARMACY SERVICES: A SPACE APPROACH}

\author{
Brígida Dias Fernandes ${ }^{\mathrm{a}^{*}}$ \\ Orcid: https://orcid.org/0000-0002-0413-8790 \\ Rodrigo Randow de Freitas ${ }^{\mathbf{b}^{* *}}$ \\ Orcid: https://orcid.org/0000-0003-0170-6892
}

\author{
Ana Carolina Melchiors ${ }^{* * *}$ \\ Orcid: https://orcid.org/0000-0002-8538-2903 \\ Roberto Pontarolo ${ }^{\mathrm{d}^{* * *}}$ \\ Orcid: https://orcid.org/0000-0002-7049-4363
}

brifernandes@gmail.com ${ }^{\mathrm{a}}$, digorandow@gmail.com ${ }^{\mathrm{b}}$, acmelchiors@gmail.com ${ }^{\mathrm{c}}$,pontarolo@ufpr.br ${ }^{\mathrm{d}}$

Universidade Federal de Minas Gerais*, Universidade Federal do Espírito Santo ${ }^{* *}$, Universidade Federal do Paraná

Data de Submissão :06/06/2019 Data de Aceite: 21/10/2019

\section{RESUMO}

Objetivo: Analisar a satisfação dos usuários com os serviços prestados em farmácias comunitárias, abordando aspectos de acessibilidade geográfica e um Sistema de Informações Geográficas. Metodologia: Foi aplicada uma versão adaptada do Questionário de Satisfação com os Serviços da Farmácia (QSSF) aos usuários de farmácias privadas localizadas no município de São Mateus/ES. A coleta de dados ocorreu próximo às farmácias, com os usuários que haviam acabado de receber os serviços e em locais públicos do município. Além da análise estatística de validação, foram criados mapas temáticos para melhor visualização dos resultados. Resultados: O QSSF adaptado foi aplicado a 530 usuários, sendo que 35\% ( $n=185)$ afirmaram terem sido atendidos pelo farmacêutico e responderam todas as questões. Após a análise fatorial, os itens originais e adaptados se organizaram em três domínios. Os usuários que afirmaram terem sido atendidos pelos farmacêuticos, entrevistados próximos às farmácias estavam mais satisfeitos do que os entrevistados em locais públicos $(4,14 \pm 0,45$ vs. $3,84 \pm 0,58 ; \mathrm{p} \leq 0,001)$. Em ambos locais de entrevistas e tipos de atendimento, as médias de satisfação relacionadas ao domínio sobre acessibilidade geográfica foram as menores. Nos locais próximos às farmácias, os atendidos pelo farmacêutico estavam mais satisfeitos do que os que não souberam quem os atendeu $(4,14 \pm 0,45$ vs. $3,83 \pm 0,47 ; \mathrm{p} \leq 0,001)$. Os mapas temáticos mostraram a concentração de farmácias nas regiões centrais, mas não houve diferença significativa entre as médias de satisfação por bairro. Conclusão: A visualização espacial dos resultados de satisfação permitiu identificar as áreas que podem se beneficiar de ações que promovam a melhoria dos serviços ofertados.

Palavras-chave: Serviços comunitários de farmácia; satisfação do paciente; análise espacial.

\section{ABSTRACT}

Objective: To analyze user satisfaction with services provided in community pharmacies, addressing aspects of geographic accessibility and using a Geographic Information System. Methodology: An adapted version of the Questionário de Satisfação com os Serviços da Farmácia (QSSF) was applied to users of private pharmacies located in São Mateus, ES, Brazil. Data collection occurred near pharmacies, with users who had just received the services and in public places in the city. In addition to the statistical validation analysis, thematic maps were created for better visualization of the results. Results: The adapted QSSF was applied to 530 users, and 35\% $(n=185)$ said they had been answered by the pharmacist and answered all questions. After factor analysis, the original and adapted items were organized into three domains. Users who claimed to have been served by pharmacists interviewed near pharmacies were more satisfied than those interviewed in public places $(4.14 \pm 0.45$ vs. $3.84 \pm 0.58 ; \mathrm{p} \leq 0.001)$. In both interview sites and types of care, the satisfaction averages related to the domain on geographical accessibility were the lowest. In locations near the pharmacies, those seen by the pharmacist were more satisfied than those who did not know who attended them $(4.14 \pm 0.45$ vs. $3.83 \pm 0.47 ; \mathrm{p} \leq 0.001)$. Thematic maps showed the concentration of pharmacies in the central regions, but there was no significant difference between the satisfaction averages per neighborhood. Conclusion: The spatial visualization of the satisfaction results allowed identifying the areas that can benefit from actions that promote the improvement of the services offered.

Keywords: Community pharmacy services; patient satisfaction; spatial analysis. 


\section{Introdução}

Serviços farmacêuticos clínicos têm sido associados a uma melhoria nos resultados em saúde dos pacientes ${ }^{1}$ e são uma das intervençõeschave para diminuição de disparidades em saúde, devendo estar estrategicamente posicionados para influenciar a saúde dos pacientes em suas vidas diárias ${ }^{2,3}$. No Brasil, os serviços farmacêuticos têm passado por modificações em seu escopo e regulação, sendo assegurado ao profissional farmacêutico a realização de atividades clínicas, organizadas em um processo de trabalho, que promovam a melhoria da qualidade de vida da população ${ }^{4-6}$.

Assim, os farmacêuticos, responsáveis técnicos por farmácias comunitárias, expandem seu papel para além da entrega de medicamento, podendo ofertar serviços clínicos, como: educação em saúde, revisão da farmacoterapia, manejo de problema de saúde autolimitado, dispensação, acompanhamento farmacoterapêutico, dentre outros $^{6}$. A realização desses serviços deve atender às necessidades em saúde dos pacientes e abrangem diferentes atividades clínicas e técnicas, respaldadas pelo Conselho Federal de Farmácia ${ }^{5}$.

Tais aspectos tornam fundamental a avaliação da qualidade dos serviços ofertados nas farmácias comunitárias, a fim de identificar lacunas e nortear o aprimoramento das intervenções. Um dos desfechos humanísticos que pode ser utilizado para avaliar os serviços prestados nas farmácias comunitárias é a satisfação do paciente ${ }^{7,8}$. A maioria das pesquisas aborda a satisfação do paciente como um construto multidimensional e utiliza questionários para avaliar os aspectos relacionados aos serviços farmacêuticos comunitários ${ }^{7}$.

Dentre os diferentes instrumentos disponíveis, o Questionário de Satisfação com os Serviços da Farmácia (QSSF) avalia a satisfação do paciente com serviços farmacêuticos orientados pela filosofia da atenção farmacêutica, considerando duas dimensões da satisfação: Manejo da Terapia e Explicação Amigável ${ }^{9,10}$. O QSSF foi originalmente elaborado por MacKeigan e Larson ${ }^{11}$ e tem sido traduzido e adaptado para diferentes idiomas ${ }^{10,12,13}$, inclusive para o português do Brasil, apresentando aspectos de validade adequados para sua utilização ${ }^{10}$.
Todavia, assim como outros instrumentos, não são abordados os aspectos relacionados à localização das farmácias e as condições de locomoção (acessibilidade geográfica) na avaliação da satisfação dos usuários ${ }^{14}$. No Brasil, não existem regras legais para abertura de farmácias baseadas em critérios geográficos e demográficos, tornando importante analisar o impacto desses aspectos na satisfação dos pacientes e no uso dos serviços farmacêuticos.

Assim, o objetivo deste trabalho foi analisar a satisfação dos usuários com os serviços prestados em farmácias comunitárias, abordando aspectos de acessibilidade geográfica utilizando um Sistema de Informações Geográficas (SIG) para melhor visualização dos resultados.

\section{Metodologia}

\section{DESENHO DO ESTUDO E COLETA DE DADOS}

Trata-se de um estudo transversal exploratório, de caráter descritivo-analítico, realizado com usuários de farmácias comunitárias localizadas no município de São Mateus/Espírito Santo. O estudo foi aprovado pelo comitê de ética em pesquisa do Centro Universitário Norte do Espírito Santo sob Parecer $n^{\circ}$ 324.102/2013 (CAAE: 17372713.9.0000.5063).

Para avaliar a satisfação do paciente, foi aplicada uma versão adaptada do QSSF ${ }^{10}$. Ao instrumento original foram incluídos cinco itens relacionados à acessibilidade geográfica, sendo quatro adaptados da primeira versão do questionário ${ }^{11}$. Os itens tratam dos fatores envolvidos na organização do serviço para que o usuário consiga utilizar o mesmo (tempo e esforço necessários para conseguir ter sua prescrição atendida, facilidade de chegar aos serviços, horário de funcionamento das farmácias). Além disso, os itens 1,2,7,9,13,17 e 20 sofreram pequenas adaptações de expressões e coloquialismos, a fim de garantir a equivalência idiomática do instrumento na população estudada.

No instrumento adaptado, os 25 itens também foram organizados para serem respondidos considerando o atendimento recebido, ou seja, se o paciente afirmasse ter sido atendido pelo 
farmacêutico responderia todas as questões. Os que não foram atendidos pelo farmacêutico, ou não souberam identificar quem os atendeu, responderiam apenas as questões relativas ao atendimento em geral e de acessibilidade. A escala de resposta utilizada pelo instrumento, do tipo Likert de 5 pontos, foi mantida.

Para caracterizaro perfil da população estudada, foram coletados dados sociodemográficos (idade, escolaridade e sexo), bem como sobre a aquisição de medicamentos (onde adquire, quantos medicamentos utiliza) e sobre acessibilidade geográfica.

A fim de evitar utilizar as informações apenas de usuários que frequentavam regularmente a mesma farmácia, a abordagem dos pacientes se deu em dois ambientes: primeiramente, foram entrevistados cinco usuários por farmácia, recrutados por amostragem de conveniência ou acessibilidade logo após terem saído do estabelecimento. Na segunda fase, entrevistou-se o quantitativo estimado de pacientes da primeira fase, considerando as 55 farmácias existentes no município no período do estudo, porém em locais públicos não vinculados às farmácias.

A entrevista foi realizada por uma equipe devidamente treinada. A coleta de dados ocorreu de agosto a novembro de 2013 e a duração da entrevista foi de aproximadamente 15 minutos, realizadas em diferentes dias da semana. A permanência em cada local público e/ou farmácia foi de cerca de três horas.

Foram incluídos usuários de farmácias privadas com idade superior a 18 anos e que não apresentassem dificuldades de compreensão por déficit cognitivo.

\section{ANÁLISE ESTATÍSTICA}

A análise estatística foi realizada com auxílio do software estatístico SPSS v17.0. Foram realizadas análises da validade de construto (consistência interna, análise fatorial, validade convergente), uma vez que foram feitas alterações na versão do instrumento já validada.

Utilizou-se o teste de Kaiser-Meyer-Olkin para análise de adequação dos dados à análise fatorial, sendo considerado valor de $\mathrm{KMO} \geq 0,8$ em geral e para todos os itens individualmente. A seguir, realizou-se a análise fatorial dos itens do instrumento utilizando a extração de componentes principais com rotação varimax, obedecendo ao critério de Kaiser do autovalor (eigenvalue) maior que 1 e o número de pontos antes da inflexão do scree plot.

A validade convergente foi realizada correlacionando-se os escores médios obtidos para cada questão do instrumento com os escores dos domínios a que pertenciam e a que não pertenciam. Foram considerados aceitáveis valores significativos de $r>0,40$.

A análise da consistência interna foi realizada pelo cálculo de a de Cronbach, sendo considerados aceitáveis valores de a superiores a 0,70. Além disso, foi analisado o impacto da retirada de cada questão ao valor de a referente ao domínio e ao escore geral. Casos em que a retirada da questão implicasse um acréscimo superior a $1 \%$ ao valor a do domínio ou geral conduziam à exclusão da pergunta do instrumento.

As médias de satisfação foram comparadas utilizando-se $\mathrm{o}$ teste $\mathrm{T}$ (independente) $\mathrm{e}$ comparadas com as variáveis categóricas por meio do teste ANOVA. O teste post-hoc de Tukey foi utilizado para identificar as correlações significativas nas variáveis com mais de duas categorias.

Para análise de correlação entre as variáveis quantitativas (médias de satisfação) foi utilizada a análise do coeficiente de correlação de Pearson. Foram considerados significativos valores de $\mathrm{p} \leq 0,001$ e intervalo de confiança de $95 \%$.

\section{ANÁLISE ESPACIAL}

Para melhor visualização dos resultados, foram criados mapas temáticos, em que os bairros da sede do munícipio foram identificados em escala de cinza, conforme o escore geral de satisfação dos serviços farmacêuticos prestados pelas farmácias presentes em cada localidade.

Foram utilizados também arquivos de imagem já georreferenciados e digitalizados do município de São Mateus, disponíveis no site do 
Instituto Jones dos Santos Neves (http://www.ijsn. es.gov.br/). O processamento das informações foi realizado pelo SIG Idrisi Selva edition (ver. 17.00- Clark University). A partir desta imagem, foi realizada a digitalização e delimitação dos bairros, farmácias e unidades de saúde, utilizando o programa CartaLinx.

O estudo obteve as coordenadas geográficas das farmácias estudadas, por meio da utilização de um aparelho de GPS (Global Positioning System). A localização das unidades de saúde foi obtida dos arquivos do Instituto Jones dos Santos Neves, que também forneceu a delimitação dos bairros, uma vez que o município não possui legislação que delimite os seus bairros. Com o intuito de identificar áreas urbanas e rurais, foi utilizada a legislação municipal que dispõe sobre o plano diretor de desenvolvimento e expansão urbana ${ }^{15}$.

\section{Resultados}

\section{ASPECTOS DE VALIDADE DO INSTRUMENTO ADAP- TADO}

Para a análise de validação do instrumento foram considerados apenas as respostas dos usuários que afirmaram terem sido atendidos pelo farmacêutico e que responderam a todos os itens. Assim, na primeira etapa foram entrevistados 255 usuários, sendo que 88 afirmaram terem sido atendidos pelo farmacêutico e responderam todas as questões. Já na segunda etapa, dos 275 usuários entrevistados, 97 afirmaram terem sido atendidos pelo farmacêutico e responderam todas as questões

A medida de Kaiser-Meyer-Olkin, demonstrou a adequação da amostra para a análise fatorial $(\mathrm{KMO}=0,907)$, sendo que os valores de comunalidades dos itens foram $\geq 0,5$. A análise inicial mostrou que cinco componentes obedeciam ao critério de Kaiser do autovalor (eigenvalue) maior que 1 e explicavam $66,73 \%$ da variância.

Entretanto, pela análise do scree plot, observouse o posicionamento de três componentes antes da inflexão. Assim, foram considerados três fatores, levando em consideração também as características dos itens do instrumento, uma vez que o instrumento original continha dois domínios e que as questões acrescentadas tratavam de itens não interpessoais. Desse modo, o primeiro componente principal explicou $39,61 \%$ da variância, o segundo $10,90 \%$ e o terceiro 7,09\%, totalizando $57,62 \%$ da variância.

Além disso, para medir a adequação de cada item nessa análise fatorial procedeu-se à análise da matriz de correlação anti-imagem, não havendo valores menores que 0,5 na diagonal. Todos os 25 itens apresentaram uma média de correlação de $0,348(-0,081-0,965)$. O a total apresentou um valor de 0,921 . O primeiro domínio (exposição agradável) constituiu-se de nove itens agrupados no componente 2 (Tabela 1 ), sendo que as questões 12 e 20 , que no instrumento original ${ }^{10}$ pertenciam ao domínio manejo da terapia, passaram para o domínio exposição agradável. A correlação entre os itens deste domínio foi de $0,461(0,296-0,751)$ e a de 0,885 .

Tabela 1. Resultado da análise fatorial do questionário de satisfação com serviços da farmácia adaptado.

\begin{tabular}{lc}
\hline \multicolumn{1}{c}{ Itens } & Componente \\
\cline { 2 - 2 } & \multicolumn{1}{c}{ Domínio 1 - Exposição Agradável } \\
\hline P2. A disponibilidade do farmacêutico em te atender? & $\mathbf{1}$ \\
P3. A relação do profissional farmacêutico com você? & 0,566 \\
P12. As orientações do farmacêutico sobre como tomar os seus medicamentos? & 0,551 \\
P14. As respostas do farmacêutico às suas perguntas? & 0,625 \\
P20. O tempo que o farmacêutico oferece para atender você? & 0,684 \\
P5. A prontidão no atendimento da sua receita? & 0,709 \\
P6. O profissionalismo dos funcionários da farmácia? & 0,777 \\
P16. A cortesia e respeito demonstrados pelos funcionários da farmácia? & 0,771 \\
P13. Os serviços da farmácia em geral? & 0,622 \\
\hline
\end{tabular}


Itens

Componente

$1 \quad 2 \quad 3$

Domínio 2 - Manejo da Terapia

P4. A habilidade do farmacêutico em avisá-lo sobre problemas que você poderia ter com seus medicamentos?

0,650

P7. A explicação do farmacêutico sobre como os seus medicamentos agem no seu organismo? $\quad 0,750$

$\begin{array}{ll}\text { P8. O interesse do farmacêutico pela sua saúde? } & 0,732\end{array}$

P9. A explicação do farmacêutico de como usar os seus medicamentos? $\quad 0,566$

P10. O empenho do farmacêutico em resolver os problemas que você tem com seus medicamentos? $\quad 0,735$

P11. A responsabilidade que o farmacêutico assume com o seu tratamento? $\quad 0,818$

P15. O empenho do farmacêutico em manter ou melhorar a sua saúde? $\quad 0,641$

$\begin{array}{ll}\text { P17. A privacidade nas conversas com farmacêutico? } & 0,552\end{array}$

P18. O empenho do farmacêutico para assegurar que os seus medicamentos façam o efeito esperado? $\quad 0,809$

P19. A explicação do farmacêutico sobre os possíveis efeitos adversos dos medicamentos? $\quad 0,836$

\section{Domínio 3- Distribuição e Acessibilidade}

P1. O profissionalismo da farmácia, quanto à infraestrutura?

P21. A distância que a farmácia fica da sua casa?

P22. O tempo que leva para chegar à farmácia?

P23. O acesso (rua, relevo e calçada) à farmácia?

P24. O horário de funcionamento da farmácia no caso de necessidade de aquisição de medicamento?

Método de extração: análise de componentes principais.

Método de rotação: varimax com normalização Kaiser. Em cada um dos componentes principais, os itens apresentando maior resultado de loading foram agrupados, dividindo o questionário em domínios.

Destaque em negrito para os itens que migraram de domínio.

O segundo domínio (manejo da terapia) foi composto por 10 itens agrupados no componente 1. A correlação entre os itens deste domínio foi de $0,577(0,414-0,792)$ e $a$ de 0,931 . Já os itens adicionados à versão do QSSF agruparam-se em um terceiro domínio, juntamente com a questão 1 , a qual pertencia ao domínio exposição agradável no original ${ }^{10}$. $\mathrm{O}$ a do novo domínio foi de 0,773 e a correlação entre os itens deste domínio foi de $0,353(0,185-0,965)$.

Quanto à validade convergente, os domínios exposição agradável e manejo da terapia apresentaram diferença na média $( \pm$
DP) das respostas $(4,23 \pm 0,51 \quad v s .3,88 \pm 0,75$; $\mathrm{p}<0,001)$. Os dois domínios se correlacionaram significativamente $(\mathrm{r}=0,70 ; \mathrm{p}<0,001)$ e tiveram boa correlação com o escore geral, sendo de $0,85(\mathrm{p}<0,001)$ para exposição agradável e 0,89 $(\mathrm{p}<0,001)$ para manejo da terapia.

Já com o domínio 3, o domínio exposição agradável apresentou uma diferença na média $( \pm \mathrm{DP})$ das respostas $(3,79 \pm 0,78$ vs. $4,23 \pm 0,51$; $\mathrm{p}<0,001)$ e uma fraca correlação $(\mathrm{r}=0,37$; $\mathrm{p}<0,001$ ), assim como ocorreu com o domínio manejo da terapia, apresentando diferença na média $(3,79 \pm 0,78$ vs. $3,88 \pm 0,75 ; \mathrm{p}<0,001)$ e uma 
fraca correlação $(r=0,29 ; . p<0,001)$. Com o escore geral, o domínio 3 apresentou uma boa correlação $(\mathrm{r}=0,63 ; \mathrm{p}<0,001)$.

Os itens do primeiro domínio apresentaram correlação na faixa de 0,30 a $0,62(\mathrm{p}<0,001)$ com o segundo domínio, de 0,12 a $0,42(\mathrm{p}<0,001)$ com o terceiro e de 0,57 a $0,82(\mathrm{p}<0,001)$ com o primeiro domínio. Os itens do segundo domínio apresentaram correlação na faixa de 0,45 a 0,68 $(\mathrm{p}<0,001)$ com o primeiro domínio, correlação de 0,12 a 0,35 ( $p>0,001)$ com o terceiro domínio e de 0,68 a $0,84(\mathrm{p}<0,001)$ com o segundo domínio. Já os itens do terceiro domínio apresentaram correlação na faixa de 0,12 a $0,30(p>0,001)$ com o primeiro domínio, de 0,13 a $0,42(\mathrm{p}>0,001)$ com o segundo domínio e de 0,50 a $0,84(\mathrm{p}<0,001)$ com o terceiro domínio.

\section{A SATISFAÇÃO DOS USUÁRIOS}

A partir dos dados de validação do instrumento, calculou-se o escore satisfação considerando o rearranjo dos itens. Não foi possível obter o escore geral do domínio 2 para o grupo que não foi atendido pelo farmacêutico ou não identificou quem os atendeu, pois tratava-se de questões relativas apenas ao atendimento farmacêutico.

Os escores médios de satisfação do grupo que afirmou ter sido atendido pelos farmacêuticos na primeira etapa foram maiores do que o grupo da segunda etapa com o mesmo atendimento (Tabela 2), apresentando diferença estatisticamente significativa entre as médias do domínio 2 $(4,17 \pm 0,53$ vs. $3,61 \pm 0,81 ; p \leq 0,001)$ e do domínio total $(4,14 \pm 0,45$ vs. $3,84 \pm 0,58 ; p \leq 0,001)$. Contudo, nas duas etapas e nos dois grupos de atendimento, os escores do domínio 1 (exposição agradável) foram os que apresentaram maiores médias e os escores do domínio 3, as menores.

Tabela 2. Escores de satisfação dos usuários com os serviços prestados em farmácias comunitárias nas duas etapas da pesquisa. São Mateus, Espírito Santo, Brasil (N=497).

\begin{tabular}{ccccc}
\hline Tipo de atendimento & Domínio 1 $( \pm$ DP) & Domínio 2 $( \pm \mathrm{DP})$ & $\begin{array}{c}\text { Domínio 3 } \\
( \pm \mathrm{DP})\end{array}$ & $\begin{array}{c}\text { Escore total } \\
( \pm \mathrm{DP})\end{array}$ \\
\hline \multicolumn{4}{c}{ Próxima às farmácias } \\
\hline $\begin{array}{c}\text { Atendidos pelos farmacêuticos }(\mathrm{n}=88) \\
\begin{array}{c}\text { Não souberam ou não foram atendidos pelos } \\
\text { farmacêuticos }(\mathrm{n}=143)\end{array}\end{array}$ & $4,33( \pm 0,42)$ & $4,17( \pm 0,53)$ & $3,82( \pm 0,80)$ & $4,14( \pm 0,45)$ \\
\hline & $4,20( \pm 0,47)$ & - & $3,58( \pm 0,62)$ & $3,83( \pm 0,47)$ \\
\hline $\begin{array}{c}\text { Atendidos pelos farmacêuticos }(\mathrm{n}=97) \\
\text { Não souberam ou não foram atendidos pelos } \\
\text { farmacêuticos }(\mathrm{n}=169)\end{array}$ & $4,13( \pm 0,58)$ & $3,61( \pm 0,81)$ & $3,76( \pm 0,76)$ & $3,83( \pm 0,58)$ \\
\hline
\end{tabular}

Ademais, na primeira etapa da pesquisa, a média geral de satisfação obtida pelo grupo atendido pelo farmacêutico foi estatisticamente diferente dos que não souberam por quem foram atendidos ou não foram atendidos pelos farmacêuticos $(4,14 \pm 0,45$ vs. $3,83 \pm 0,47 ; p \leq 0,001)$.
A partir da média de escores de satisfação geral obtida pelas farmácias de cada bairro na primeira etapa, foi construído um mapa temático da sede do munícipio. Na Figura 1 está a avaliação dos usuários que disseram terem sido atendidos pelo farmacêutico, sendo que a maioria dos bairros apresentou média de satisfação próxima de 5 . 
Figura 1. Mapa temático da sede do município de São Mateus/ES segundo o escore de satisfação geral obtido no grupo de usuários que afirmou ter sido atendido pelos farmacêuticos na primeira etapa da pesquisa.

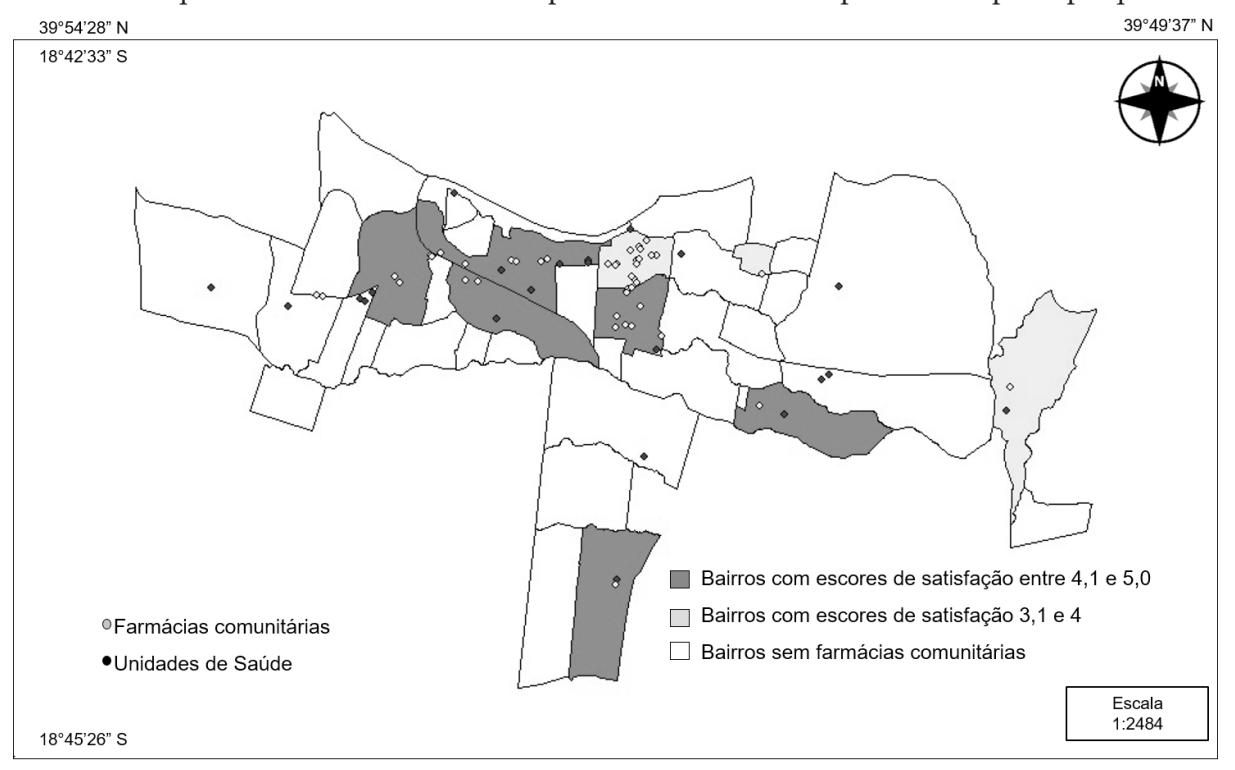

As farmácias concentravam-se em bairros centrais do município, sendo que dos 38 bairros pertencentes à sede de São Mateus, apenas dez possuíam farmácias. No bairro localizado no centro do município também foi onde houve maior número de pacientes que disseram ter sido atendidos pelo farmacêutico $(29,5 \% ; n=26)$, na primeira etapa da pesquisa. Contudo, quanto às médias de satisfação, não houve diferença significativa entre os bairros, sendo que nas zonas afastadas do centro, os bairros que apresentavam apenas uma farmácia também apresentaram altos escores de satisfação.

Para o mapa gerado com os escores obtidos pela avaliação dos pacientes que disseram não terem sido atendidos ou que não sabiam por quem foram atendidos na primeira etapa é possível visualizar que a maioria dos bairros apresentou escores de satisfação mais baixos (Figura 2).

Figura 2. Mapa temático da sede do munícipio de São Mateus/ES segundo o escore de satisfação geral obtido no grupo de usuários que não sabia por quem havia sido atendido na primeira etapa da pesquisa.

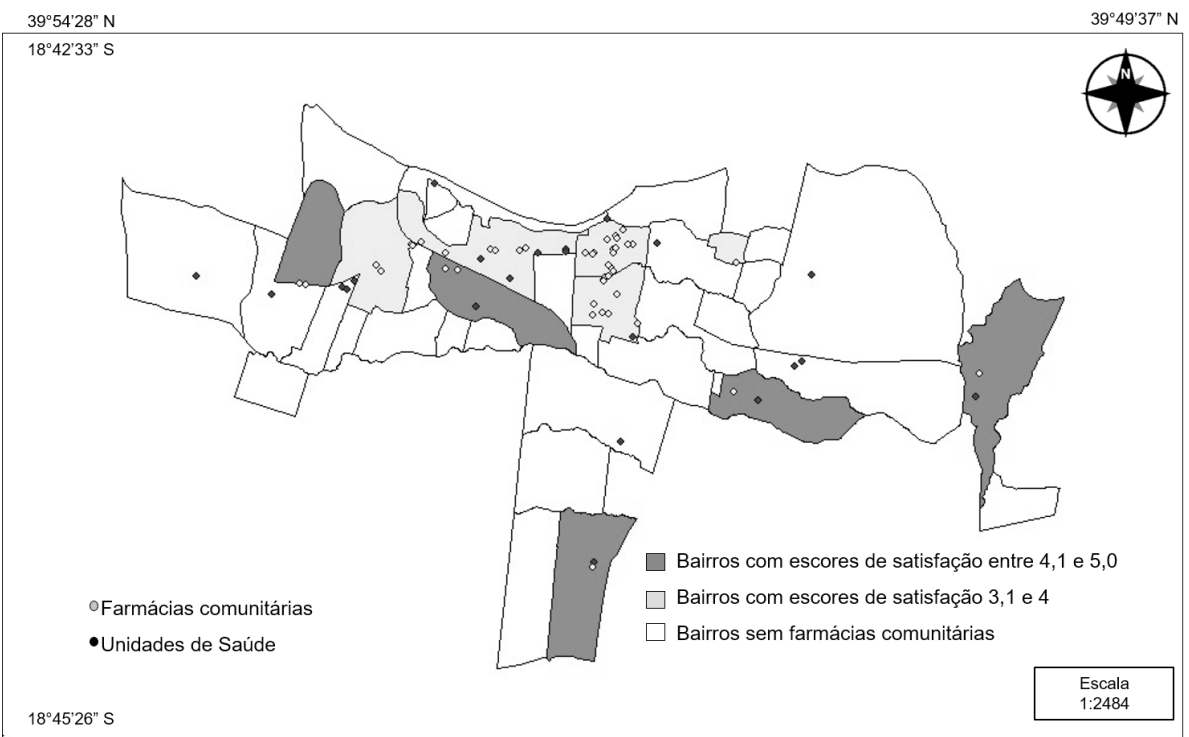




\section{PERFIL DOS PACIENTES}

O perfil dos entrevistados nas duas etapas foi semelhante, sendo a maioria pertencente ao sexo feminino, dentro da faixa etária de 20 a 59 anos e com segundo grau completo (Tabela 3). A escolha de comprar sempre no mesmo estabelecimento foi relatado pela maioria dos usuários, em ambos os grupos e locais de entrevista.

Em relação à acessibilidade à farmácia, os usuários também se locomovem mais frequentemente a pé até as farmácias e residem no mesmo bairro que os estabelecimentos avaliados.

Tabela 3. Perfil dos usuários que participaram da pesquisa de satisfação na primeira e segunda etapa. São Mateus, Espírito Santo, Brasil (N=497).

\begin{tabular}{|c|c|c|c|c|}
\hline \multirow{2}{*}{ Variáveis } & \multicolumn{2}{|c|}{ Próximo às farmácias } & \multicolumn{2}{|c|}{ Locais públicos } \\
\hline & $\mathrm{AF}(\mathrm{n}=88)$ & NA $(n=143)$ & $\mathrm{AF}(\mathrm{n}=97)$ & NA $(n=169)$ \\
\hline \multicolumn{5}{|l|}{ Faixa etária } \\
\hline 18 a 20 anos & $8,0 \%(7)$ & $3,5 \%(5)$ & $26,8 \%(26)$ & $8,9 \%(15)$ \\
\hline 20 a 29 anos & $14,8 \%(13)$ & $28,0 \%(40)$ & $34,0 \%(33)$ & $42,0 \%(71)$ \\
\hline 30 a 39 anos & $17,0 \%(15)$ & $26,6 \%(38)$ & $18,6 \%(18)$ & $16,0 \%(27)$ \\
\hline 40 a 49 anos & $22,7 \%(20)$ & $18,2 \%(26)$ & $8,2 \%(8)$ & $12,4 \%(21)$ \\
\hline 50 a 59 anos & $15,9 \%(14)$ & $11,9 \%(17)$ & $4,1 \%(4)$ & $11,8 \%(20)$ \\
\hline 60 a 69 anos & $14,8 \%(13)$ & $9,1 \%(13)$ & $7,2 \%(7)$ & $5,3 \%(9)$ \\
\hline 70 a 79 anos & $5,7 \%(5)$ & $1,4 \%(2)$ & $1,0 \%(1)$ & $3,6 \%(6)$ \\
\hline Mais de 80 anos & $1,1 \%(1)$ & $0,7 \%(1)$ & 0 & 0 \\
\hline \multicolumn{5}{|l|}{ Sexo } \\
\hline Feminino & $64,8 \%(57)$ & $53,1 \%(76)$ & $60,8 \%(59)$ & $59,2 \%(100)$ \\
\hline Masculino & $35,2 \%(31)$ & $46,9 \%(67)$ & $39,2 \%(38)$ & $40,8 \%(69)$ \\
\hline \multicolumn{5}{|l|}{ Escolaridade } \\
\hline Analfabeto & $4,5 \%(4)$ & $2,1 \%(3)$ & $1,0 \%(1)$ & $0,6 \%(1)$ \\
\hline $1^{\circ}$ Grau Incompleto & $25 \%(22)$ & $13,3 \%(19)$ & $8,2 \%(8)$ & $9,5 \%(16)$ \\
\hline $1^{\circ}$ Grau Completo & $11,4 \%(10)$ & $15,4 \%(22)$ & $4,1 \%(4)$ & $8,9 \%(15)$ \\
\hline $2^{\circ}$ Grau Incompleto & $12,5 \%(11)$ & $7,7 \%(11)$ & $12,4 \%(12)$ & $3,6 \%(6)$ \\
\hline $2^{\circ}$ Grau Completo & $29,5 \%(26)$ & $38,5 \%(55)$ & $33,0 \%(32)$ & $35,5 \%(60)$ \\
\hline $3^{\circ}$ Grau Incompleto & $8,0 \%(7)$ & $10,5 \%(15)$ & $28,9 \%(28)$ & $31,4 \%(53)$ \\
\hline $3^{\circ}$ Grau Completo & $9,1 \%(8)$ & $12,6 \%(18)$ & $12,4 \%(12)$ & $10,7 \%(18)$ \\
\hline \multicolumn{5}{|c|}{ Sempre compram na mesma farmácia } \\
\hline Sim & $69,3 \%(61)$ & $51,7 \%(74)$ & $64,9 \%(63)$ & $55,0 \%(93)$ \\
\hline Não & $30,7 \%(27)$ & $47,6 \%(68)$ & $35,1 \%(34)$ & $45,0 \%(76)$ \\
\hline \multicolumn{5}{|c|}{ Também adquirem medicamentos pelo SUS } \\
\hline Sim & $62,5 \%(55)$ & $48,9 \%(70)$ & $54,6 \%(53)$ & $43,8 \%(74)$ \\
\hline \multicolumn{5}{|c|}{ Residem no mesmo bairro da farmácia avaliada } \\
\hline Sim & $48,9 \%(43)$ & $50,3 \%(72)$ & $35,1 \%(34)^{*}$ & $35,5 \%(60)^{*}$ \\
\hline Não & $48,9 \%(43)$ & $46,2 \%(66)$ & $36,1 \%(35)^{\star}$ & $32,5 \%(55)^{*}$ \\
\hline Em outra cidade & $2,3 \%(2)$ & $1,4 \%(2)$ & $1,0 \%(1)^{\star}$ & $1,8 \%(3)^{\star}$ \\
\hline
\end{tabular}




\begin{tabular}{|c|c|c|c|c|}
\hline \multirow{2}{*}{ Variáveis } & \multicolumn{2}{|c|}{ Próximo às farmácias } & \multicolumn{2}{|c|}{ Locais públicos } \\
\hline & $\operatorname{AF}(n=88)$ & NA $(n=143)$ & $\operatorname{AF}(n=97)$ & NA $(n=169)$ \\
\hline \multicolumn{5}{|c|}{ Locomoção até a farmácia } \\
\hline A pé & $53,4 \%(47)$ & $55,9 \%(80)$ & $64,9 \%(63)$ & $64,5 \%(109)$ \\
\hline Automóvel próprio & $21,6 \%(19)$ & $24,5 \%(35)$ & $18,6 \%(18)$ & $20,1 \%(34)$ \\
\hline Transporte público & $15,9 \%(14)$ & $7,7 \%(11)$ & $10,3 \%(10)$ & $9,5 \%(16)$ \\
\hline Táxi & 0 & $0,7 \%(1)$ & 0 & $0,6 \%(1)$ \\
\hline Outros (bicicleta) & $9,1 \%(8)$ & $11,2 \%(16)$ & $6,2 \%(6)$ & $5,3 \%(9)$ \\
\hline \multicolumn{5}{|c|}{ Média de medicamentos em uso } \\
\hline & $2,63( \pm 1,77)$ & $2,55( \pm 1,92)$ & $2,20( \pm 1,58)$ & $2,34( \pm 1,81)$ \\
\hline
\end{tabular}

AF: Usuários que relataram terem sido atendidos pelo farmacêutico. NA: Usuários não atendidos pelo farmacêutico ou que não sabiam por quem foram atendidos.

*Dados calculados apenas quando os usuários informavam o bairro de residência e da farmácia avaliada.

\section{Discussão}

A satisfação do usuário é um desfecho em saúde importante, porém de difícil medição, pois os serviços possuem características exclusivas, além de serem intangíveis, visto que a sua produção ocorre concomitantemente ao seu consumo ${ }^{16,17}$, tornando a avaliação extremamente subjetiva, pois tenta-se capturar o grau de satisfação com o atendimento recebido, que não pode ser conhecido por meio da simples observação direta do atendimento ${ }^{18}$. Dessa forma, é essencial que a avaliação da satisfação considere as evidências de validade dos instrumentos utilizados.

Nesse sentido, o presente estudo contribui com evidências de validade de uma versão adaptada do QSSF que inclui aspectos de acessibilidade geográfica. A correlação entre os itens dos domínios originais e os valores de a de Cronbach foram mais baixos do que os encontrados no estudo de tradução e adaptação do instrumento para o Brasil ${ }^{10}$, porém maiores que os do domínio 3 , formado por questões relativas à acessibilidade. Estes valoresindicam quehá melhor confiabilidade, bem como validade de construto, em relação aos domínios que são diretamente relacionados à interação do paciente com farmacêutico.

Outro estudo mostra que questões não relacionadas diretamente aos aspectos interpessoais, como acessibilidade e conveniência, apresentam menor consistência interna ${ }^{19}$. Entretanto, a adição de tais questões foi importante para conhecer a opinião dos usuários sobre a distribuição e acessibilidade das farmácias. Além disso, nenhuma questão quando retirada implicou um acréscimo superior a $1 \%$ ao valor a do domínio ou escore geral.

No instrumento adaptado também houve rearranjo do item 12, relacionado as orientações do farmacêutico sobre como tomar os medicamentos e do 20, sobre o tempo que o farmacêutico leva para atender, que pertenciam ao domínio manejo da terapia no instrumento adaptado para o Brasil $^{10}$. Enquanto o item 1, sobre a aparência profissional da farmácia, que pertencia ao domínio de exposição agradável ${ }^{10}$ migrou para o domínio 3. Alterações na distribuição dos itens também foi verificada nos estudos de adaptação do instrumento original para outras línguas ${ }^{10,12}$, sugerindo que as populações dos estudos têm percepções diferentes sobre os aspectos relacionados ao manejo da terapia, considerando a orientação sobre como utilizar o medicamento e o tempo que o farmacêutico oferece para atender como atividades "tradicionais", as quais estão habituados. Tais alterações também podem estar relacionadas às modificações nas expressões utilizadas nos itens, tornando necessário mais 
estudos para identificar qual versão dos itens possui maior clareza de linguagem para população brasileira.

As médias de satisfação dos que afirmaram terem sido atendidos por farmacêuticos na entrevista, logo após saírem da farmácia, foram maiores do que os entrevistados em locais públicos, apresentando diferença estatisticamente significativa entre as médias do domínio relacionado ao manejo da terapia. Esses dados confirmam que o momento (local) onde se realiza a entrevista interfere na avaliação dos indivíduos, sendo mais críticos aqueles cujas entrevistas foram realizadas em locais distantes dos estabelecimentos avaliados e, aqueles com melhor percepção geral, os que foram abordados logo após receberem o serviço, pois possivelmente visitam tais farmácias regularmente, podendo ter maior afinidade com os profissionais e serviços avaliados ${ }^{20,21}$.

Em relação ao grupo entrevistado logo após o atendimento na farmácia, identificou-se que os usuários estavam mais satisfeitos quando foram atendimentos pelo profissional farmacêutico, o que pôde ser visualizado nos mapas temáticos. Contudo, os dados revelam que a maioria dos usuários não identifica os farmacêuticos, talvez porque este profissional não possua fácil identificação ou porque não está presente na área de atendimento. Embora seja uma exigência legal ${ }^{22}$, na maioria das farmácias comunitárias estudadas a identificação dos farmacêuticos é ausente ${ }^{23}$.

Independentemente do local de entrevista e do tipo de atendimento, as médias de satisfação do domínio 3 foram as menores. Assim, verifica-se uma maior insatisfação com aspectos relacionadas às condições de infraestrutura, bem como a distância da residência até a farmácia, o tempo gasto no percurso e condições das vias de acesso. Tais aspectos dos serviços de saúde, incluindo farmácias comunitárias, podem ser barreiras na utilização dos mesmos pelos usuários. Em um estudo qualitativo realizado com pacientes portadores de deficiência física, auditiva e visual sobre a acessibilidade aos serviços de saúde, um terço dos pacientes relatou o tempo necessário para chegar até o serviço como uma dificuldade de acessibilidade $^{24}$. Já em estudo realizado na Nova Zelândia, identificou-se que quando o tempo de viagem era longo até os serviços de saúde, as visitas às farmácias eram menos frequentes ${ }^{25}$.

Contudo, não houve diferença significativa entre as médias de satisfação dos serviços por bairros. Em uma das pesquisas que aborda a satisfação dos pacientes com acessibilidade às farmácias comunitárias americanas verificouse que a maioria dos entrevistados não relatou problemas com o acesso à farmácia mesmo sendo de áreas urbanas ou suburbanas ${ }^{14} \mathrm{e}$, de forma semelhante, em outro estudo a maioria dos entrevistados estava satisfeito com a localização da farmácia, sendo elas de rede ou independentes ${ }^{26}$. Dessa forma, torna-se necessário mais estudos que avaliem o impacto da distribuição e organização geográfica das farmácias comunitárias na satisfação e em outros desfechos de saúde dos pacientes.

É importante ressaltar que esses altos escores de satisfação podem não traduzir a qualidade do serviço farmacêutico, pois são influenciados pelos padrões subjetivos do usuário e o seu referencial de qualidade. As limitações do estudo também estão relacionadas à utilização de um instrumento que avalia aspectos de serviços farmacêuticos reorientados pela filosofia da atenção farmacêutica, realidade que pode ainda não ser a vivenciada na área de estudo, o que levou a modificações na estrutura dos itens e do instrumento. Contudo, as análises de validade contribuem com a discussão sobre adequabilidade do questionário em populações brasileiras.

\section{Conclusão}

O presente estudo contribui com a discussão sobre as evidências de validade de uma versão adaptada do QSSF que aborda questões de acessibilidade geográfica às farmácias comunitárias. Ademais, a visualização espacial dos resultados de satisfação permitiu identificar as áreas que podem se beneficiar de ações que promovam a melhoria dos serviços ofertados.

Os resultados dessa pesquisa também destacam uma preocupação com área de serviços farmacêuticos prestados em farmácias comunitárias, uma vez que a maioria dos 
pacientes não sabe ou não são atendidos pelos farmacêuticos, sendo, possivelmente, privados de um importante serviço de saúde.

\section{Referências}

1. Melchiors AC, Correr CJ, Venson R, Pontarolo R. An analysis of quality of systematic reviews on pharmacist health interventions. Int J Clin Pharm. 2012;34(1):32-42.

2. Mullins CD, Blatt L, Gbarayor CM, Yang H-WK, Baquet C. Health disparities: A barrier to high-quality care. Am J Heal Pharm. 2005;62(18):1873-82.

3. Schommer JC, Singh RL, Cline RR, Hadsall RS. Market dynamics of community pharmacies in Minnesota. Res Soc Adm Pharm. 2006;2(3):347-58.

4. Brasil. Lei ${ }^{\circ} 13.021$, de 8 de agosto de 2014. Dispõe sobre o controle sanitário do comércio de drogas, medicamentos, insumos farmacêuticos e correlatos. Diário Oficial da União, Poder Executivo, Brasília, DF, 11 ago. 2014a. Seção 1, p. 1, Edição Extra.

5. Conselho Federal de Farmácia (Brasil). Resolução $\mathrm{n}^{\circ}$. 585, de 29 de agosto de 2013. Regulamenta as atribuições clínicas do farmacêutico e dá outras providências. Diário Oficial da União, Poder Executivo, Brasília, DF, 25 set. 2013f. Seção 1, p. 186-188.

6. Conselho Federal de Farmácia (Brasil). Serviços farmacêuticos diretamente destinados ao paciente, à família e à comunidade: contextualização e arcabouço conceitual. Brasília: Conselho Federal de Farmácia; 2016. $200 \mathrm{p}$.

7. Naik Panvelkar P, Saini B, Armour C. Measurement of patient satisfaction with community pharmacy services: a review. Pharm World Sci. 2009 Oct 9;31(5):525-37.

8. Olave Quispe SY, Traverso ML, Palchik V, García Bermúdez E, La Casa García C, Pérez Guerrero MC, et al. Validation of a patient satisfaction questionnaire for services provided in Spanish community pharmacies. Int J Clin Pharm. 2011;33(6):949-57.

9. Larson LN, Rovers JP, MacKeigan LD. Patient satisfaction with pharmaceutical care: update of a validated instrument. J Am Pharm Assoc. 2002;42(1):44-50.

10. Correr CJ, Pontarolo R, Melchiors AC, Souza RA de $\mathrm{P}$ e, Rossignoli P, Fernández-llimós F. Satisfação dos usuários com serviços da farmácia: tradução e validação do Pharmacy Services Questionnaire para o Brasil. Cad Saude Publica. 2009;25(1):87-96.

11. MacKeigan LD, Larson LN. Development and validation of an instrument to measure patient satisfaction with pharmacy services. Med Care. 1989;27(5):522-36.

12. Iglésias P, Santos HJ, Fernández-llimós F, Fontes E, Leal M, Monteiro C. Tradução e Validação do "Pharmacy Services Questionnaire" para Português (europeu). Seguimento Farmacoter. 2005;3(1):43-56.

13. Kim KI, Suh HS, Kwak A, Kim S, Han N, Lee E, et al. Development and validation of a modified Korean version of the Pharmacy Services Questionnaire (PSQ-K) for the quality assessment of community pharmacy services. PLoS One. 2017;12(4):e0174004.

14. Malewski DF, Ream A, Gaither CA. Patient satisfaction with community pharmacy: Comparing urban and suburban chain-pharmacy populations. Res Soc Adm Pharm. 2015 Jan;11(1):121-8.

15. Prefeitura Municipal de São Mateus. Lei Municipal n. ${ }^{\circ}$ 007, de 10 de dezembro de 2004. Dispõe sobre o plano diretor de desenvolvimento e expansão urbana do município de São Mateus e dá outras providências.

16. Donabedian A. Evaluating the Quality of Medical Care. Milbank Q. 2005;83(4):691-729.

17. Schommer JC, Kucukarslan SN. Measuring patient satisfaction with pharmaceutical services. Am J Heal Pharm. 1997;54:2721-32.

18. Ware JE, Snyder MK, Wright WR, Davies AR. Defining and measuring patient satisfaction with medical care. Eval Program Plann. 1983;6(3-4):247-63.

19. Mendonça KMPP, Guerra RO. Desenvolvimento e validação de um instrumento de medida da satisfação do paciente com a fisioterapia. Rev Bras Fisioter. 2007;11(5):369-76.

20. Esperidião M, Trad LAB. Avaliação de satisfação de usuários. Cien Saude Colet. 2005;10:303-12.

21. Wirth F, Tabone F, Azzopardi LM, Gauci M, ZarbAdami M, Serracino-Inglott A. Consumer perception of the community pharmacist and community pharmacy services in Malta. J Pharm Heal Serv Res. 2010;1(4):18994.

22. Agência Nacional de Vigilância Sanitária (Brasil). Resolução RDC nº. 44, de 17 de agosto de 2009. Dispõe sobre boas práticas farmacêuticas para o controle sanitário do funcionamento, da dispensação e da comercialização de produtos e da prestação de serviços farmacêuticos em farmácias e drogarias e dá ou.

23. Fernandes BD. Avaliação da qualidade dos serviços farmacêuticos prestados em farmácias comunitárias e a satisfação dos usuários: uma análise espacial. Universidade Federal do Paraná; 2014. 
24. Castro SS, Lefèvre F, Lefèvre AMC, Cesar CLG. Acessibilidade aos serviços de saúde por pessoas com deficiência. Rev Saude Publica. 2011;45(1):99-105.

25. Hiscock R, Pearce J, Blakely T, Witten K. Is Neighborhood Access to Health Care Provision Associated with Individual-Level Utilization and Satisfaction? Health Serv Res. 2008;43(6):2183-200.

26. Briesacher B, Corey R. Patient satisfaction with pharmaceutical services at independent and chain pharmacies. Am J Heal Pharm. 1997;54(5):531-6.

\section{Como citar este artigo:}

Fernandes BD, Freitas RR, Melchiors AC, Pontarolo R. Satisfação dos usuários com serviços da farmácia comunitária: uma abordagem espacial. Rev. Aten. Saúde. 2019; 17(62): 12-23. 